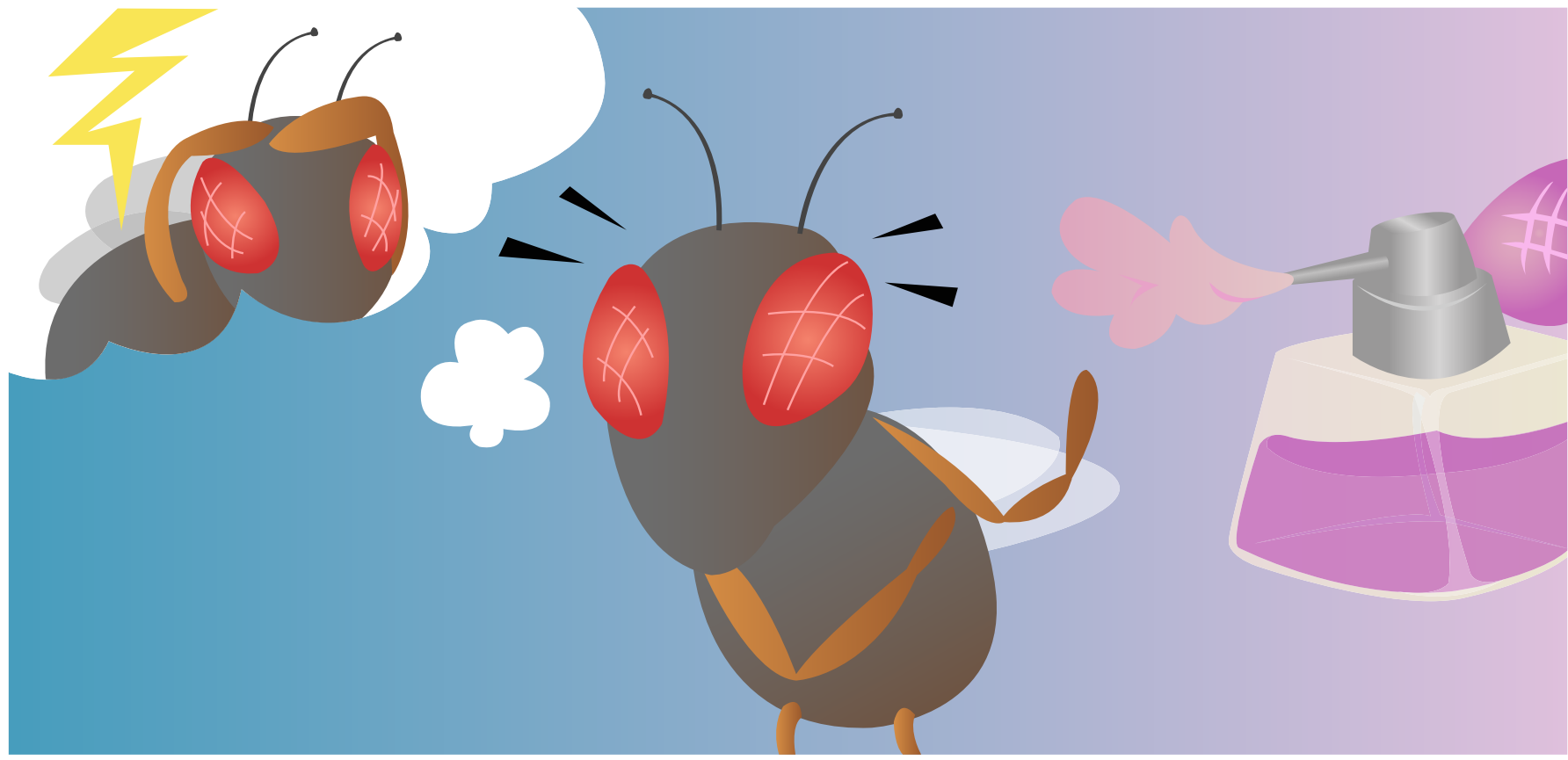

\title{
FRUIT FLIES CAN TEACH US HOW WE FORGET
}

\section{Isaac Cervantes-Sandoval* and Ronald L. Davis}

Department of Neuroscience, The Scripps Research Institute Florida, Jupiter, FL, United States

\section{REVIEWED BY: \\ NEW YORK \\ TIMES \\ STUDENT \\ JOURNEYS}

AGE: 8-11
Have you ever wondered, "why do we forget?" Can you imagine a life where you could remember everything? Although this sounds interesting, it might not be a good idea after all. Scientists have started to learn that forgetting is very important for the normal functioning of the brain. If you cannot erase memories that you do not need, you could have problems learning new things or focusing your attention on the things that are important. Using fruit flies to study learning and forgetting, we discovered a protein named Scribble that is very important for forgetting odor memories. Yes, as surprising as it may seem, these small flies can learn and forget. We will explain this in the article below.

\section{HOW AND WHY DO WE FORGET?}

We all know what forgetting is; however, have you ever asked yourself, "why do we forget?" We can easily imagine a world where we remember every detail and we might think how wonderful that would be. But, we can also reason that maybe remembering everything could present a problem. You must have forgotten at least once-probably many times-to do your homework or make 
your bed. You have surely forgotten, every now and then, to brush your teeth. We all forget certain things. Usually, we tend to think that forgetting is bad and undesirable because forgetting important things can cause problems. Forgetting your homework can get you into trouble with your teachers and parents and if you forget to brush your teeth too many times, this will mean more trips to the dreaded dentist! We also link forgetting with brain diseases such as Alzheimer's disease or senile dementia. These are diseases of the brain that cause difficulty remembering recent events (called short-term memory loss). These are serious brain diseases in which forgetting too many things causes problems for the person with the disease. However, we also know that forgetting has some very important advantages for brain activity, mental and emotional health, and even survival.

But why do we forget? Let us think about this in terms of a computer. Suppose you have a computer with 10,000 pictures that you have saved over the past 2 years, but after looking at the pictures once or twice, you never opened them again. Now, suppose you need a funny picture of your pet cat or dog to make a birthday card for your friend. You know you have that picture somewhere among the 1,000 of pictures, but unless you have organized your 10,000 pictures really well by name, you will have to check picture by picture until you locate the one that you want. This will take a long time, maybe a few days, and you might not even find it before your friend's birthday! Also, keeping so many pictures will fill up the memory of your computer and if you need to save new pictures there will be no more space for them. Additionally, saving so many files in the memory of your computer will make it slow, because it has to deal with so much information. Now imagine a second situation, in which the computer is designed to delete pictures that have not been re-opened within a month from the last time that you looked at them. This would cause the pictures that are not re-opened frequently to be erased, but those that are re-opened would be re-saved every time they are looked at. This would reduce the clutter to perhaps only 100 files on the computer. This smaller group of pictures contains only those that are really important to you. In this second situation, it would be much easier to find the photo of your pet dog or cat, perhaps taking only a few minutes and allowing you to make the birthday card before your friend's birthday passes! The speed of your computer will be fast and you will still have enough space to store new pictures.

A similar process happens when memories are stored in your brain. Can you imagine what would happen if you remembered everything that you ate every day of your life? If you ate three meals a day, in 10 years you would remember over 10,000 meals! This is pretty useless information that would waste your brain resources and affect your brain functions in a bad way. So, forgetting is important for saving the important information from our everyday experiences and deleting the useless information.

So, forgetting has its advantages, and it is a normal process in our brains. However, until recently, we did not know how the brain decides what 


\section{DNA}

Deoxyribonucleic acid is the chemical that carries the genetic information in the cells of all living forms; this information is used in the growth, development, functioning, and reproduction.

\section{PROTEIN}

Proteins are large molecules composed by long chains of amino acids. Proteins are very important and perform multiple tasks in all living organisms. These tasks range from making the structures that form the cells to accelerate most chemical reactions in that occur inside all cells. information to remember and what information to forget, or how the brain accomplishes forgetting. It was thought that memories simply faded or were deleted naturally over time.

\section{FRUIT FLIES CAN LEARN, AND JUST LIKE US, THEY FORGET}

Neuroscientists, scientists who study how the brain works, have been very interested in learning how memories are stored in the brain. However, neuroscientists have only very recently begun to study forgetting. To understand how the human brain forgets, we can study how fruit flies forget. Fruit flies are awesome, small insects that are great for scientific research. They grow very fast in the laboratory and we can produce as many flies as we want. Their genetic material, or DNA, is also very easy to change. DNA is a very long, thin chemical that contains the instructions to build any living organism. DNA contains genes, which are sections of the DNA that tell a cell how to make a protein. The instructions contained in the DNA of the flies can be changed in the lab. Genes can be removed, making a mutant fly. In this way, we can explore what happens to a fly if a piece of these instructions is removed.

Flies also have small brains that are much easier to explore than a human or mouse brain. Although you will find this surprising, human brains and fruit fly brains have many things in common. Amazingly, fruit flies can learn simple tasks, they can form memories, and they can also forget, just as we do. Just like human brains, fly brains are made up mainly of cells called neurons. Neurons are the cells that transmit information across the brain. Groups of neurons, like a computer, can form circuits that process and store information.

To study how flies forget, we first teach the flies a simple task. Then we give the flies a test to see how much they remember and how much they have forgotten. What do we teach them and how do we test their memory? Flies have a very powerful sense of smell. For this reason, we give the flies odors (or different smells) to learn. We first allow the flies to smell an odor and at the same time we give them a mild shock of electricity. This causes the flies to learn that they will feel a bit of pain when they smell the odor. Then we test how much the flies remember by placing the flies in a tiny area with the odor that they smelled when they were shocked. If they remember well, the flies run away from the odor, thinking they will be shocked again-in this case, they score an $\mathrm{A}+$ on this test. If they have forgotten, and do not run away, they score an F. Normally, flies will remember very well and will get an A if they are tested a few minutes after learning. But if there is long time between learning and testing, which for a fruit fly is 1 day, they will forget and get an F (Figure 1).

Using this simple experiment, we and other neuroscientists have found a new group of neurons in the fly brain that form a circuit in charge of making new 


\section{FIGURE 1}

Fruit flies can learn simple tasks, they form memories, and they can also forget. During the learning session, flies are allowed to smell an odor and at the same time they receive a mild shock of electricity. Flies learn that they will feel a bit of pain when they smell the odor. Then, memory is tested to see how much the flies remember by placing the flies in a tiny area with the odor that they smelled during the learning session. If they remember well, the flies run away from the odor and they score an A. If they have forgotten and do not run away from the odor, they score an $\mathrm{F}$.

\section{DOPAMINE}

An important molecule in the brain that functions as a neurotransmitter; that means dopamine is a chemical that is used by neurons to communicate one to another. It is known to be very important for the pleasurable sensation of rewards but also for the aversive sensation of punishments.

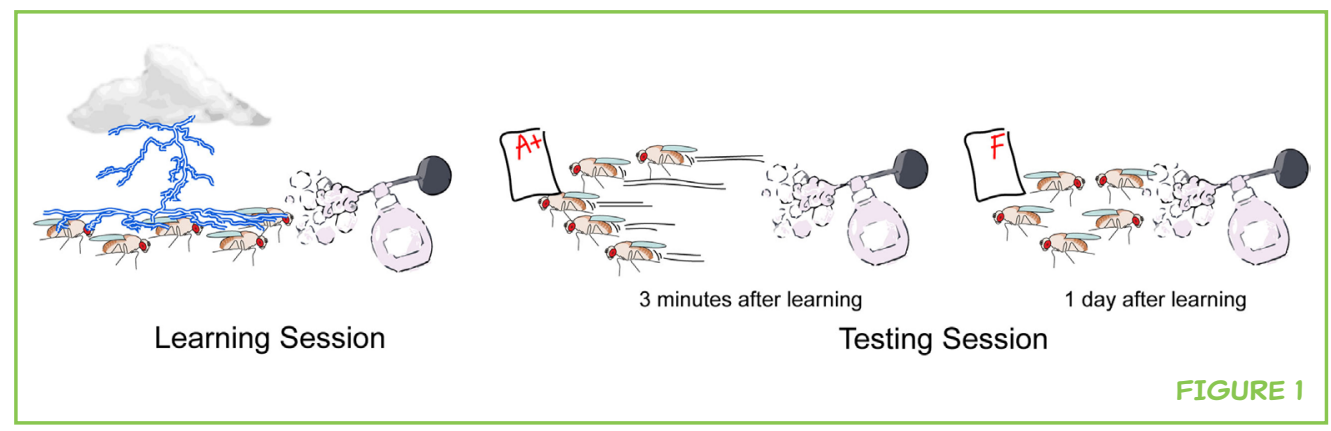

memories. These neurons work together to learn and store a new memory. Once the memory is stored, these same neurons continue to work and this begins to slowly erase the memory that was just formed. If the new memories are not important, they will be gradually eliminated by the activity of this circuit, until they are completely erased [1]. However, if the information learned is really important-like the location of a new source of food-or if the memory is recalled an hour or two later, then the memory will be "protected" from forgetting. The neurons that cause forgetting use a brain molecule called dopamine. Interestingly, mutant flies that do not have one of the genes responsible for interpreting the dopamine signal, called dopamine receptor, remember the odor they have learned for a very long time. Basically, these flies have a long-lasting memory because they cannot forget. Another gene that is very important for forgetting unimportant memories is named Rac. The Rac gene makes a protein that speeds up changes to the skeleton of most cells. It is thought that changes in the skeleton of neurons are very important to create the structures that hold new memories. Rac speeds up the chemical reactions that undo these changes in the skeleton and in doing so causes forgetting [2].

\section{SCRIBBLE PROTEIN SUPPORTS THE FORGETTING MACHINERY}

We wanted to find out what other molecules inside the cell control how flies forget. To accomplish this goal, we changed thousands of fly genes and then tested the flies with odors and electric shock to see how well they remembered or forgot. We found that when we removed a gene called Scribble, flies could still remember very well 1 day after learning. That told us that these flies had awesome memories, because normal flies cannot remember anything 1 day after the learning session (Figure 2). We then tested whether the learning of new information would cause the forgetting of an old memory. Flies and humans both forget older memories when new information interferes with them. In other words, new memories erase old memories. To test whether Scribbleless flies (flies in which Scribble was deleted) have problems updating memories, we trained flies to associate odor A with getting an electric shock and then, after some time passed, we trained the same flies to associate the shock with a different, unrelated odor (odor B). Normal flies usually learn very easily that 


\section{FIGURE 2}

Top, flies in which the Scribble gene is intact produce a scaffolding protein that allows for normal forgetting of odor memories. Bottom, flies in which the Scribble gene was removed could still remember the odor very well one day after learning. This flies had amazing memory, because normal flies cannot remember anything 1 day after the learning session.

\section{FIGURE 3}

The Scribble scaffolding protein supports Rac and this in turn helps control how the fly forgets. Scribble is like the metal scaffolding that supports Rac and Rac is like an eraser that will be in charge of deleting memories.

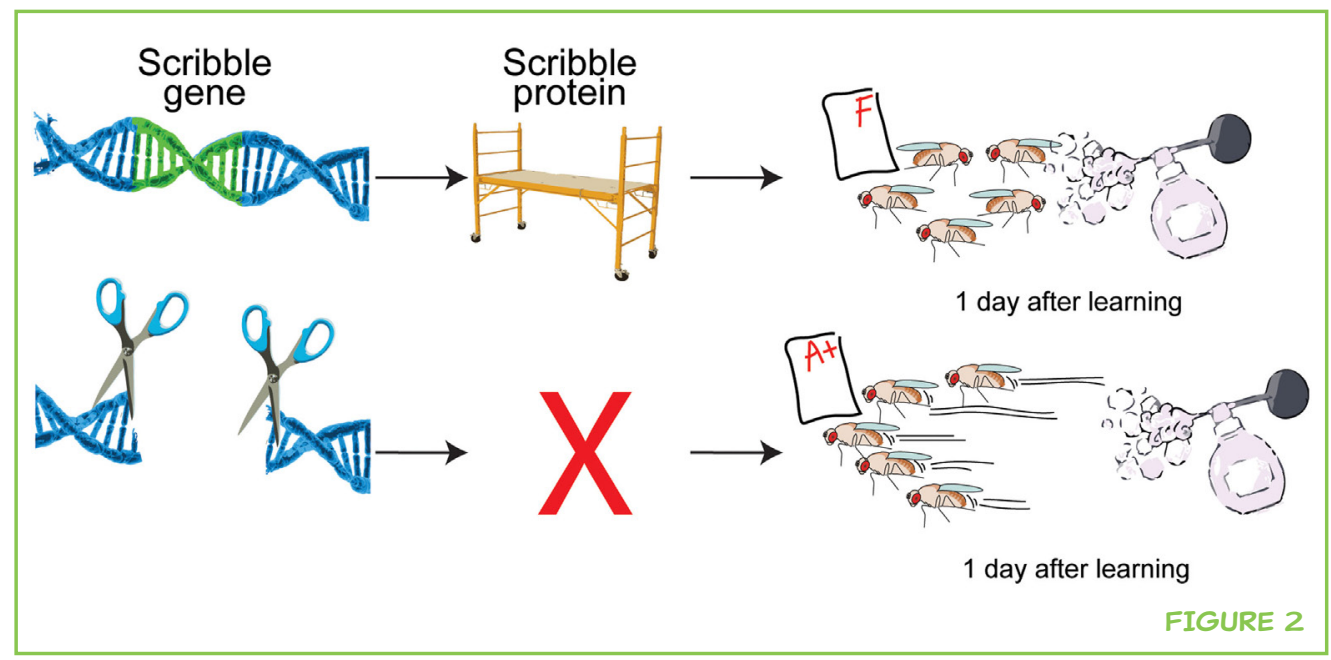

FIGURE 3

odor $\mathrm{B}$ is painful, but this new memory makes them forget the memory that odor A is also painful. In contrast, Scribleless flies remembered odor A just as well as if they had never been trained to odor B-they did not forget odor A. We also found that, because these Scribbleless flies cannot forget, it was harder for them to make new memories. The unforgotten memories prevented new memories from being made. In this way, we confirmed the idea that the removal of old memories is necessary to free up space for new memories.

This gene is called Scribble because eggs of flies that do not have this gene have a skin that looks like a scribble. The Scribble gene makes a scaffolding (or framework) protein. Have you ever seen how a new building is built? The new construction is usually surrounded by a metal structure called scaffolding that 
SCAFFOLD

PROTEIN

Specific types of proteins that are known for their ability to interact with other proteins and in this way orchestrate many complex processes.

serves as a support, so that workers can step on it and make the structure of the new building. Scaffolding proteins work in cells in the same way-they support many other proteins in the cell by sticking to them and putting together groups of different proteins. They act like your skeleton does to give support and shape to your body. Scaffolding proteins also support and give shape to cells. We discovered that the Scribble protein is important for forgetting because it attaches to the protein Rac that we talked about earlier [3]. Scribble supports Rac and this, in turn, helps control how the fly forgets. So in summary, Scribble is like the metal scaffolding that supports Rac and Rac is like an eraser that is in charge of deleting memories (Figure 3). Although all these findings were discovered using an experiment that asked flies to remember a smell, it is possible that the genes we discovered in this experiment are also involved in creating other kinds of memories, not just memories of smell-but that has not been tested yet.

\section{CONCLUSION}

Scaffolding proteins like Scribble, because they can bind to multiple proteins at the same time, are important to the function of cells. In our research, we found that Scribble interacts with the Rac protein to regulate the very important brain process of forgetting. These are among the first discoveries that will help scientists understand how fly brains forget. These findings in flies will also help us understand why and how humans forget. Remember, while flies and humans are different, we also have many things in common. For example, humans also have Scribble and Rac proteins in the brain. Perhaps these proteins are the first, necessary building blocks that will help scientists eventually understand the many different human brain disorders that cause increased forgetting and memory loss.

\section{ORIGINAL SOURCE ARTICLE}

Cervantes-Sandoval, I., Chakraborty, M., MacMullen, C., and Davis, R. L. 2016.

Scribble scaffolds a signalosome for active forgetting. Neuron 90:1230-42. doi:10.1016/j.neuron.2016.05.010.

\section{REFERENCES}

1. Berry, J., Cervantes-Sandoval, I., Nicholas, E., and Davis, R. 2012. Dopamine is required for learning and forgetting in Drosophila. Neuron 74:530-72. doi:10.1016/j. neuron.2012.04.007

2. Shuai, Y., Lu, B., Hu, Y., Wang, L., Sun, K., and Zhong, Y. 2010. Forgetting is regulated through Rac activity in Drosophila. Cell 140(4):579-89. doi:10.1016/j. cell.2009.12.044 
3. Cervantes-Sandoval, I., Chakraborty, M., MacMullen, C., and Davis, R. L. 2016. Scribble scaffolds a signalosome for active forgetting. Neuron 90(6):1230-42. doi:10.1016/j.neuron.2016.05.010

SUBMITTED: 06 June 2017; ACCEPTED: 23 November 2017; PUBLISHED ONLINE: 12 December 2017.

EDITED BY: Sabine Kastner, Princeton University, United States

CITATION: Cervantes-Sandoval I and Davis RL (2017) Fruit Flies Can Teach Us How We Forget. Front. Young Minds 5:63. doi:10.3389/frym.2017.00063

CONFLICT OF INTEREST STATEMENT: The authors declare that the research was conducted in the absence of any commercial or financial relationships that could be construed as a potential conflict of interest.

COPYRIGHT @ 2017 Cervantes-Sandoval and Davis. This is an open-access article distributed under the terms of the Creative Commons Attribution License (CC BY). The use, distribution or reproduction in other forums is permitted, provided the original author(s) or licensor are credited and that the original publication in this journal is cited, in accordance with accepted academic practice. No use, distribution or reproduction is permitted which does not comply with these terms.

\section{REVIEWED BY}

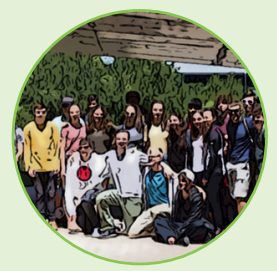

\section{NEW YORK TIMES STUDENT JOURNEYS, AGE: 8-11}

We are a group of high school students from the United States traveling with New York Times Student Journeys in quest of gaining a deeper understanding of science and storytelling. We are interested in a range of scientific topics and hope to broaden our knowledge base about ways in which science can be shared in the world.

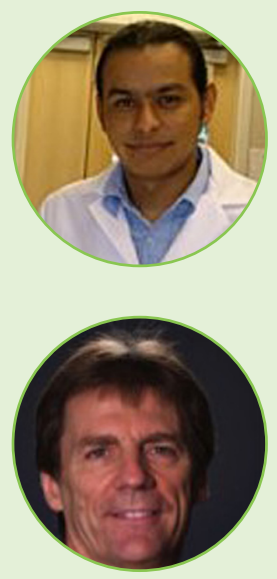

\section{AUTHORS}

\section{ISAAC CERVANTES-SANDOVAL}

I am Isaac Cervantes-Sandoval; I was born in Mexico where I got my Ph.D. in Biology. I am interested in the study of neuroscience, particularly how brain stores information and how this information is then forgotten. Understanding how and why do we forget is the main goal of my research. *isandova@scripps.edu

\section{RONALD L. DAVIS}

I am the Founding Chairman of the Department of Neuroscience at the Scripps Research Institute Florida. I am a leading expert on the biology of memory formation and the brain disorders that disrupt memory. My current biomedical research focuses on the biology of memory formation, forgetting, bipolar disorder, and the development of therapeutics for Alzheimer's disease and related disorders. 